\title{
Calibration of PIXE yields using binary thin films on $\mathrm{Si}$
}

\author{
J. Meersschaut ${ }^{\mathrm{a}, *}$, J. Carbonel $^{\mathrm{a}}$, M. Popovici ${ }^{\mathrm{a}}$, Q. Zhao $^{\mathrm{b}}$, A. Vantomme ${ }^{\mathrm{b}}$, W. Vandervorst ${ }^{\mathrm{a}, \mathrm{b}}$ \\ ${ }^{a}$ Imec, Kapeldreef 75, B-3001 Leuven, Belgium \\ b IKS, KU Leuven, Celestijnenlaan 200D, B-3001 Leuven, Belgium
}

\section{A R T I C L E I N F O}

Article history:

Received 30 September 2013

Received in revised form 10 March 2014

Accepted 10 March 2014

Available online 26 April 2014

\section{Keywords:}

PIXE

Calibration

Thin films

\begin{abstract}
A B S T R A C T
We describe the use of binary thin films on $\mathrm{Si}$ to calibrate the yields in proton-induced X-ray emission (PIXE) measurements. Besides of the element to be calibrated, the standards also contain a common reference element. The incorporation of a common reference element allows one to eliminate errors in the accumulated beam charge during the calibration of the PIXE set-up. The binary calibration standards allow us to determine the response function with an accuracy close to $1 \%$. As an example, we will perform the calibration for $\mathrm{Fe}$ and $\mathrm{Co}$, and we will determine the Co concentration in $\mathrm{Fe}_{1-x} \mathrm{Co}_{x}$ thin films.
\end{abstract}

(c) 2014 Elsevier B.V. All rights reserved.

\section{Introduction}

Ion beam techniques are recognized as versatile methods for probing the composition and the thickness of thin films. In particular, Rutherford backscattering spectrometry (RBS) is an established technique for the standard less determination of the areal density and depth profile of elements in thin films. Yet, in certain cases, the information accessible via Rutherford backscattering spectrometry is limited due to signal interference from elements with a small mass difference. In those cases, proton-induced $\mathrm{X}$-ray emission (PIXE) spectroscopy may prove a valuable complementary technique, as it has a much better elemental resolving power $[1,2]$. The usefulness of the PIXE technique to determine the composition of multi-elemental thin films was demonstrated on various systems $[3,4]$. For thin film systems, the limit of detection (LOD) for PIXE is comparable to the sensitivity reached with RBS [5].

The X-ray yield measured from a thin film scales with the accumulated beam charge, the ionization cross section, the fluorescence yield, the filter attenuation, and the detector efficiency [1]. Since not all the values are easily known with a sufficient absolute accuracy, the response function of the experimental set-up is usually derived from measurements on calibration standards. After an extended calibration procedure, the accuracy amounts to $~ 5 \%$ $[6-8]$.

In the present work, we describe the use of thin films on Si for determining the response function of the PIXE set-up. The

\footnotetext{
* Corresponding author. Tel.: +32 16288592 .

E-mail address: Johan.Meersschaut@imec.be (J. Meersschaut).
}

standards are qualified by Rutherford backscattering spectrometry measurements. The standards consist of binary thin films: besides of the element to be calibrated, they also contain a common reference element. The latter allows one to eliminate errors in the accumulated beam charge during the PIXE measurement. Thus, the accuracy in the stoichiometry determination of thin films is improved to $\sim 1 \%$. Alternatively, the approach may be used to refine the databases for ionization cross-section and fluorescence yields.

\section{Experimental details}

The RBS experiments are performed in a random rotation mode using $1.5 \mathrm{MeV} \mathrm{He}^{+}$ions from the $6 \mathrm{SDH}$ tandem accelerator (National Electrostatics Corporation). The end-station is a 5-axis goniometer [9]. The angle between the sample normal and the beam is $10^{\circ}$. The solid angle of the PIPS detector is $0.42 \mathrm{msr}$, the scattering angle is $170^{\circ}$. The beam spot is confined to $1 \times 1 \mathrm{~mm}^{2}$, the current is limited to $25 \mathrm{nA}$. In-house developed analysis software is used to fit the spectra and to deduce the areal density. The influence of screening is treated to the first order by using a screened Coulomb cross section obtained by multiplying the scattering cross section by a correction factor $F=\left(1-0.049 Z_{1} Z_{2}^{4 / 3} / E\right)$ with $E$ given in keV [10].

The PIXE measurements are performed at the 5SDH-2 Pelletron. The $\mathrm{H}^{+}$source is of the SNICS type (National Electrostatics Corporation) using a $\mathrm{TiH}_{2}$ target as the cathode. The angle between the sample normal and the beam is set to $10^{\circ}$. The X-ray detector for the PIXE measurements is a $30 \mathrm{~mm}^{2}$ HPGe detector (Canberra) located at $45^{\circ}$ from the incoming beam. The target detector distance is $120 \mathrm{~mm}$. The absorber in front of the detector is a 
$52 \mu \mathrm{m}$ thick Mylar foil. The energy calibration of the spectra is realized using a ${ }^{57} \mathrm{Co}$ radioactive source that emits characteristic $\mathrm{X}$-rays at $6.40 \mathrm{keV}$ and gamma rays at $14.40 \mathrm{keV}$. The GUPIX software is used to analyze the spectra.

The RBS and the PIXE experiments were performed at different instruments. Indeed, the calibration procedure outlined below does not require that the RBS and PIXE measurements be performed in the same tool or at the same time. Thus, universal PIXE standards will become available that may be exchanged between different laboratories and institutes.

\section{PIXE calibration}

\subsection{Calibration using thin film standards}

For thin films, the intensity $I(e l)$ of the X-ray line for an element $(e l)$ is related to the areal density of the element $A(e l)$ through the equation:

$I(e l)=Q \cdot \Omega \cdot \varepsilon(e l) \cdot t(d, e l) \cdot Y_{1}(e l) \cdot A(e l)$.

$Q$ is the collected charge, $\Omega$ is the solid angle of the detector, $\varepsilon$ is the efficiency of the detector, $t$ is the transmission through the $\mathrm{X}$-ray absorber of thickness $d$, and $Y_{1}$ is the X-ray yield of the element in counts per unit charge per unit solid angle. To be able to determine $A_{\mathrm{PIXE}}(e l)$ from the intensity $I(e l)$ measured in an X-ray spectrum, one needs to determine the value of the different parameters in Eq. (1).

The efficiency of the detector is computed using models as detailed in reference [1]. The X-ray yield for a given element may be computed using databases on the ionization cross-section, the fluorescence yield, and on Coster-Kronig transitions. One of the limiting factors in the absolute accuracy of PIXE analysis is the uncertainty on the ionization cross section [11]. In addition, it proves difficult to determine to a very high accuracy the values of $\Omega$-the effective solid angle of the detector, $\varepsilon$-the energy dependent efficiency of the detector, $Q$-the collected charge for a given experiment, and $d$-the thickness of the X-ray absorber.

Following the implementation of the GUPIX software package [12], the parameters concerning the geometry of the set-up and the efficiency of the charge measurement are gathered in an instrumental constant $H$, defined as $H=f_{Q} \cdot \Omega$, where $f_{Q}$ is a factor to convert an experimental to absolute accumulated charge. Further, it is recommended to calibrate the thickness of the absorber (d) using a known sample. Finally, the GUPIX software has the option to account for an apparent energy dependence of the response function.

A detailed calibration of a PIXE set-up is commonly achieved using thin film standards. After calibration of the set-up using certified MicroMatter reference samples, the PIXE results typically show a dispersion of approximately $5 \%$ [6-8].

\subsection{Calibration using thin films on Si substrates characterized with RBS}

Rutherford backscattering spectrometry is a versatile technique to characterize the areal density of a thin film. The accumulated charge during the RBS experiment is derived from the signal height of the substrate in combination with accurate literature values of the stopping power [13]. The absolute accuracy of Rutherford backscattering spectrometry for the determination of quantity of material in thin films on $\mathrm{Si}$ is estimated around 1\% [14]. Therefore, thin films deposited on a silicon substrate, qualified through RBS, are well suited standards for the calibration of the PIXE set-up.

Consider a thin film of element (el) deposited on a Si substrate. Let us denote $A_{\mathrm{RBS}}(e l)$ the areal density of the element characterized by RBS, and $A_{\mathrm{PIXE}}(e l)$ the areal density of the element determined by PIXE using the nominal value of $\Omega$ as input for $H$. The elementspecific correction factor is

$H_{e l}=\Omega \cdot \frac{A_{\mathrm{PIXE}}(e l)}{A_{\mathrm{RBS}}(e l)}$.

The uncertainty on the correction factor is the combined uncertainty of the uncertainty on the areal density determined by RBS (1\%) and the uncertainty as a result of the experimental non-reproducibility of the charge-collection measurement. Therefore, the combined uncertainty on $\mathrm{H}$ is estimated to $\sim 3 \%$.

\subsection{Calibration using binary thin films}

To optimize the accuracy in the composition determination by PIXE, one must minimize the uncertainty on the ratio of the correction factors for various elements. We deduce the ratio of the correction factors from standards that contain both the element of interest $(e l)$ as well as a reference element (ref). We define the ratio of the correction factors as $h_{e l / r e f}=H_{e l} / H_{r e f}$. From Eq. (2), it follows that

$h_{e l / r e f}=\frac{A_{\mathrm{PIXE}}(e l)}{A_{\mathrm{PIXE}}(r e f)} \cdot \frac{A_{\mathrm{RBS}}(r e f)}{A_{\mathrm{RBS}}(e l)}$.

For both PIXE and RBS, the areal densities are deduced from a single spectrum. Therefore, the uncertainties on the accumulated charge and on the detector solid angle do not propagate to the uncertainty on the $h$-value. Rather, the accuracy of $h$ is mainly limited by counting statistics (peak intensity, background) in both RBS and PIXE, by the validity of the Rutherford scattering cross sections in RBS, and by the effects of secondary fluorescence in PIXE.

\section{Calibration of the PIXE yield for Fe and Co}

We will illustrate the procedure to determine the response function of the PIXE set-up for the case of Fe and Co. However, it should be noted that a calibration standard should not consist of a combination of elements with close atomic number, because of interfering signals in RBS. To overcome the signal interference in RBS, if one needs to determine $h_{\mathrm{Fe} / \mathrm{Co}}$ with Fe and Co having a small mass difference, one may use the transitive property: $h_{\mathrm{Fe} / \mathrm{Co}}=$ $h_{\mathrm{Fe} / r e f} \cdot h_{\text {ref/Co }}$. Because of the availability of Pt in our deposition tool, we choose Pt as a reference element, first to obtain $h_{\mathrm{Fe} / \mathrm{Pt}}$ and $h_{\mathrm{Co} / \mathrm{Pt}}$, and then to calculate $h_{\mathrm{Fe} / \mathrm{Co}}=h_{\mathrm{Fe} / \mathrm{Pt}} / h_{\mathrm{Co} / \mathrm{Pt}}$.

The calibration of the iron and cobalt relative yields has been realized using samples consisting of $\mathrm{Fe}(15 \mathrm{~nm}) / \mathrm{Pt}(20 \mathrm{~nm}) / \mathrm{SiO}_{2}$ $(100 \mathrm{~nm}) / \mathrm{Si}$-substrate and $\mathrm{Co}(20 \mathrm{~nm}) / \mathrm{Pt}(20 \mathrm{~nm}) / \mathrm{SiO}_{2}(100 \mathrm{~nm}) /$ Si-substrate. The RBS spectrum for the iron-platinum sample is presented in Fig. 1(a). The contributions from the different elements in the sample are identified in the RBS spectrum. The results of the analysis for the areal density is $A_{\mathrm{RBS}}(\mathrm{Fe})=124.6 \pm$ $3.5 \times 10^{15} \mathrm{at} / \mathrm{cm}^{2}$ and $A_{\mathrm{RBS}}(\mathrm{Pt})=124.9 \pm 2.5 \times 10^{15} \mathrm{at} / \mathrm{cm}^{2}$. The uncertainties on the screening correction are such that the error in the absolute value of the cross-section for Pt may be as much as $0.5 \%$, while for the light elements it may be neglected [15]. The uncertainties on the reported areal densities include the statistical as well as the estimated systematic uncertainties.

The PIXE spectrum obtained on the calibration standard Fe $(15 \mathrm{~nm}) / \mathrm{Pt}(20 \mathrm{~nm}) / \mathrm{SiO}_{2} \quad(100 \mathrm{~nm}) / \mathrm{Si}$-substrate is shown in Fig. 1(b). The red curve illustrates the contributions from the various elements as derived from the GUPIX analysis. The K-lines of Fe (resp. Co, not shown here) are well-separated from the signal of Pt, and the elements are probed with a good statistical accuracy. From the peak intensities, one may determine the areal density for $\mathrm{Fe}$ (resp. Co) and Pt in the calibration samples. The element-specific 
(a)

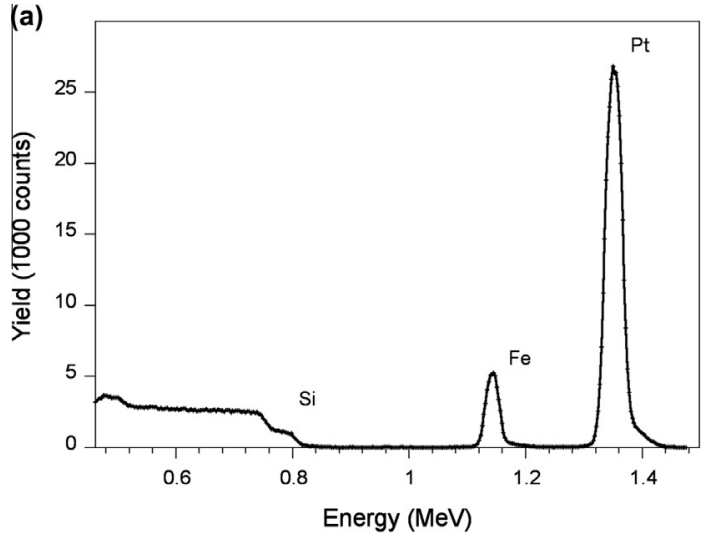

(b)

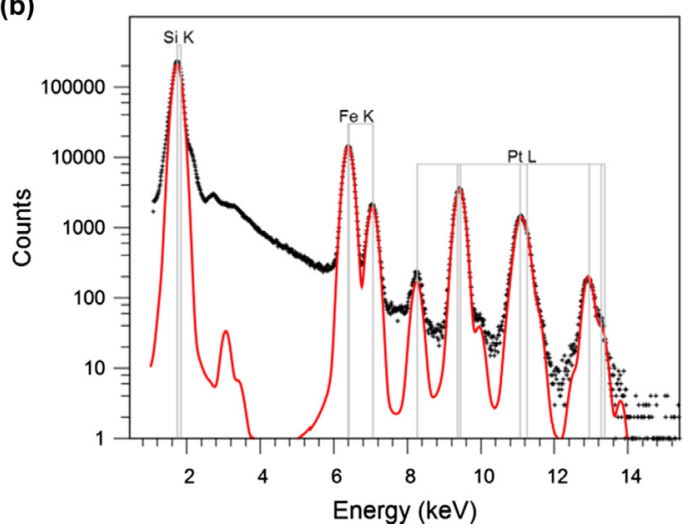

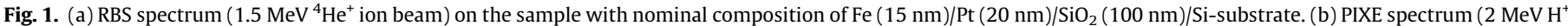

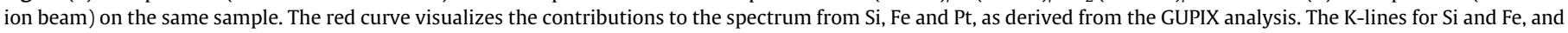
the L-lines for Pt are indicated. (For interpretation of the references to color in this figure legend, the reader is referred to the web version of this article.)

calibration factors $\left(H_{\mathrm{Fe}}, H_{\mathrm{Co}}\right.$, and $\left.H_{\mathrm{Pt}}\right)$ obtained through comparison between RBS and PIXE are listed in Table 1.

The element-specific correction factors $\left(H_{e l}\right)$ account for the inaccuracies in the detector efficiency, the charge collection efficiency, the fluorescence coefficient, etc. A good agreement between the $H_{\mathrm{Pt}}$-values is obtained between the two samples. One notices a significant difference between the $H$-values obtained at $2 \mathrm{MeV}$ and $2.5 \mathrm{MeV}$, attributed to the difficulty to quantitate the accumulated charge. The uncertainty on the $H$-values is, at best, $3 \%$. If the areal density of multiple elements is determined and corrected using the above-mentioned $\mathrm{H}$-factors, then each element is determined with an accuracy of $\sim 3 \%$. Thus, the uncertainty on the ratio of the elements readily amounts to $\sim 6 \%$.

A more beneficial approach is to use the instrumental ratios of correction factors $\left(h_{e l / r e f}\right)$. Table 1 shows the $h$-factor for Fe-Pt and Co-Pt. Combining the values of $h_{\mathrm{Fe} / \mathrm{Pt}}$ and $h_{\mathrm{Co} / \mathrm{Pt}}$, one obtains $h_{\mathrm{Fe} / \mathrm{Co}}=(1.000 \pm 0.008)$, i.e. practically no correction is needed. The value $h_{\mathrm{Fe} / \mathrm{Co}_{0}}$ being close to unity indicates that the relative $\mathrm{X}$-ray yield of iron and cobalt is very well modeled in the GUPIX software (note that the absolute difference in X-ray yield amounts to $30 \%)$. In contrast, the values of $h_{\mathrm{Fe} / \mathrm{Pt}}$ and $h_{\mathrm{Co} / \mathrm{Pt}}$ differ significantly from unity. The uncertainty in the detector efficiency and in the absorber thickness cannot solely explain the observed effect. Rather, the imperfect modeling of the X-ray yield is suspected to be the main source of deviation from unity. Indeed, it was pointed out that the databases for ionization cross-sections and fluorescence yields of L-lines need further refinements [16-18].

The reported uncertainty on $h_{\mathrm{Fe} / \mathrm{Co}}$ illustrates the benefit of calibrating the PIXE set-up in terms of relative efficiencies by means of binary thin films. Remarkably many uncertainties cancel out for the calculation of the instrumental ratios. In particular, the error on $h_{\mathrm{Fe} / \mathrm{Co}}$ as a result of the uncertainty on the RBS screening correction reduces to the equivalent RBS uncertainty for the same elements; the uncertainty on the screening correction for $\mathrm{Pt}$ cancels out for the calculation of $h_{\mathrm{Fe} / \mathrm{Co}}$. Finally, secondary fluorescence in PIXE is to be considered. Secondary fluorescence effects are believed to be negligible for the present calibrations [19], since the energy difference between the Pt L-lines and the Fe- (resp. Co-) $\mathrm{K}$-lines is large. Besides, secondary fluorescence effects are much reduced in thin films as compared to bulk materials.

\section{Characterization of FeCo thin films}

The applicability of PIXE to determine the Fe/Co ratio has been demonstrated before [4]. Here, we illustrate the use of the instrumental sensitivity ratio to determine the $\mathrm{Fe} / \mathrm{Co}$ atomic ratio. The PIXE spectra obtained with a $2 \mathrm{MeV}$ proton beam for the FeCosamples with a nominal thickness of $0.2 \mathrm{~nm}$ and $1.2 \mathrm{~nm}$ are shown in Fig. 2. The X-ray lines for $\mathrm{Si}, \mathrm{Fe}$ and $\mathrm{Co}$ are superimposed on a slowly varying background from the Bremsstrahlung. The $\mathrm{Fe} \mathrm{K}_{\beta}$ and $\mathrm{Co}_{\alpha}$ cannot be identified separately, since the energy difference between them is smaller than the intrinsic resolution of the detection system. The Co $\mathrm{K}_{\beta}$ line may be recognized visually in the spectrum for the $1.2 \mathrm{~nm}$ thick FeCo sample.

The areal density for a series of samples with varying $\mathrm{FeCo}$ thickness is reported in Table 2. We observe a fair agreement between the RBS and PIXE results. The differences between RBS and PIXE are mainly attributed to the difficulty to measure the accumulated charge for the PIXE measurement.

The PIXE results on the Fe/Co atomic ratio is reported in Table 2. The composition is not accessible from conventional RBS measurements because of signal interference between the elements. It is observed that PIXE allows to determine the $\mathrm{Fe} / \mathrm{Co}$ atomic ratio with an accuracy of $3 \%$ for $1.2 \mathrm{~nm}$ thick films. Since the instrumental ratio of correction factors $\left(h_{e l / r e f}\right)$ is known to an accuracy better than $1 \%$, the uncertainty in the composition mainly stems from the counting statistical uncertainty. Since cobalt appears to be

Table 1

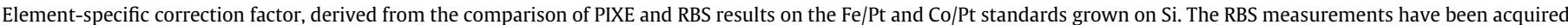
using a $\mathrm{He}^{+}$ion beam of $1.5 \mathrm{MeV}$. The energy of the $\mathrm{H}^{+}$ion beam used for the PIXE measurements are indicated in the table.

\begin{tabular}{|c|c|c|c|c|}
\hline & & $H_{\mathrm{Fe}}$ & $H_{\mathrm{Pt}}$ & $h_{\mathrm{Fe} / \mathrm{Pt}}$ \\
\hline $\mathrm{Fe}(15 \mathrm{~nm}) / \mathrm{Pt}(20 \mathrm{~nm})$ & $2 \mathrm{MeV} \mathrm{H}^{+}$ & $(2.72 \pm 0.08)^{*} 10^{-4}$ & $(2.45 \pm 0.06)^{*} 10^{-4}$ & $1.108 \pm 0.007$ \\
\hline \multirow[t]{2}{*}{$\mathrm{Fe}(15 \mathrm{~nm}) / \mathrm{Pt}(20 \mathrm{~nm})$} & $2.5 \mathrm{MeV} \mathrm{H}^{+}$ & $(2.27 \pm 0.06)^{*} 10^{-4}$ & $(2.05 \pm 0.05)^{*} 10^{-4}$ & $1.108 \pm 0.007$ \\
\hline & & $\mathrm{H}_{\mathrm{Co}}$ & $\mathrm{H}_{\mathrm{Pt}}$ & $h_{\mathrm{Co} / P t}$ \\
\hline Co $(20 \mathrm{~nm}) / \mathrm{Pt}(20 \mathrm{~nm})$ & $2 \mathrm{MeV} \mathrm{H}^{+}$ & $(2.75 \pm 0.07)^{*} 10^{-4}$ & $(2.48 \pm 0.06)^{*} 10^{-4}$ & $1.109 \pm 0.007$ \\
\hline Co $(20 \mathrm{~nm}) / \mathrm{Pt}(20 \mathrm{~nm})$ & $2.5 \mathrm{MeV} \mathrm{H}^{+}$ & $(2.30 \pm 0.06)^{*} 10^{-4}$ & $(2.07 \pm 0.05)^{*} 10^{-4}$ & $1.107 \pm 0.006$ \\
\hline
\end{tabular}



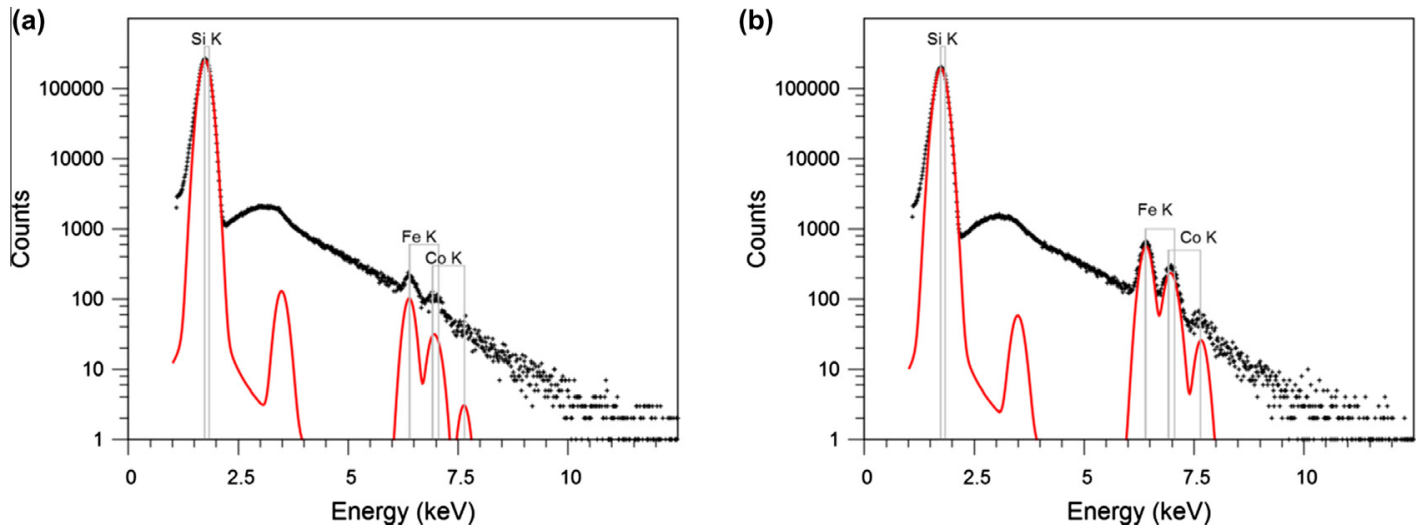

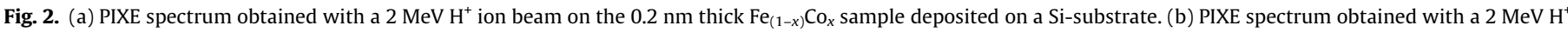

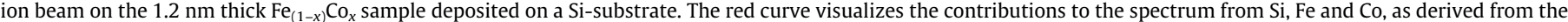

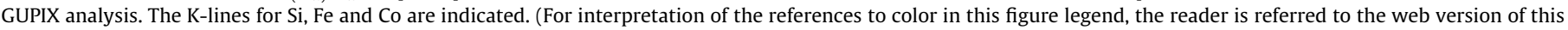
article.)

Table 2

Results of the areal density for samples of varying nominal FeCo-thickness obtained with RBS $\left(1.5 \mathrm{MeV}^{4} \mathrm{He}^{+}\right)$and PIXE $\left(2.0 \mathrm{MeV} \mathrm{H}^{+}\right)$ion beam. The atomic ratio of $\mathrm{Fe} / \mathrm{Co}$ is only accessible from the PIXE analysis.

\begin{tabular}{llll}
\hline $\begin{array}{l}\text { Nominal } \\
(\mathrm{nm})\end{array}$ & $\begin{array}{l}A_{\mathrm{RBS}}(\mathrm{Fe}+\mathrm{Co}) \\
\left(10^{15} \mathrm{at} / \mathrm{cm}^{2}\right)\end{array}$ & $\begin{array}{l}A_{\text {PIXE }}(\mathrm{Fe}+\mathrm{Co}) \\
\left(10^{15} \mathrm{at} / \mathrm{cm}^{2}\right)\end{array}$ & $\begin{array}{l}\mathrm{Fe} / \mathrm{Co} \text { atomic } \\
\text { ratio }\end{array}$ \\
\hline 0.2 & 1.25 & 1.22 & $3.75 \pm 0.75$ \\
0.4 & 2.48 & 2.77 & $3.06 \pm 0.28$ \\
0.8 & 4.99 & 5.29 & $2.61 \pm 0.13$ \\
1.2 & 7.44 & 8.12 & $2.50 \pm 0.08$ \\
\hline
\end{tabular}

the least abundant element in the films, it contributes most to the uncertainty on the composition.

\section{Conclusion}

We have calibrated the response function of the PIXE set-up for the determination of iron and cobalt. The standards are binary thin films deposited on $\mathrm{Si}$. The binary calibration standards allow us to determine the response function with an accuracy of approximately $1 \%$. As an application, we have used the calibration to determine the Co concentration in $\mathrm{Fe}_{1-x} \mathrm{Co}_{x}$ thin films.

\section{Acknowledgments}

This work was supported by the Fund for Scientific ResearchFlanders (FWO), the KU Leuven Concerted Action (GOA/09/006), the Belgian Interuniversity Attraction Poles research programs (IAP P6/42), and by the FP7 SPIRIT project (Contract No. 227012). We are grateful to M.A. Reis for helpful discussions.

\section{References}

[1] Particle-induced X-ray emission spectrometry (PIXE), in: S.A.E. Johansson, J.L. Campbell, K.G. Malmqvist (Eds.), Chemical Analysis: A Series of Monographs on Analytical Chemistry and Its Applications, vol. 133, John Wiley \& Sons, NY, 1995.

[2] J.L. Campbell, Particle-induced X-ray emission: PIXE, in: Y. Wang, M. Nastasi (Eds.), Handbook of Modern Ion Beam Materials Analysis, Second ed., Materials Research Society, Warrandale, PA, 2009. Chapter 11.

[3] M.A. Reis, N.P. Barradas, C. Pasual-Izarra, P.C. Chaves, A.R. Ramos, E. Alves, G. Gonzales-Aguilar, M.E.V. Costa, I.M. Miranda Salvado, Nucl. Instr. Meth. B 261 (2007) 439.

[4] N.P. Barradas, R. Mateus, M. Fonseca, M.A. Reis, K. Lorenz, I. Vickridge, Nucl. Instr. Meth. B 268 (2010) 1829.

[5] J. Meersschaut, M. Käyhkö, H.P. Lenka, T. Witters, Q. Zhao, A. Vantomme, W. Vandervorst, AIP Conf. Proc. 1525 (2013) 190-194, http://dx.doi.org/10.1063/ 1.4802317.

[6] W. Maenhaut, H. Raemdonck, Nucl. Instr. Meth. B 1 (1984) 123.

[7] A. Shariff, K. Bülow, M. Elfman, P. Kristiansson, K. Malmqvist, J. Pallon, Nucl. Instr. Meth. B 189 (2002) 131.

[8] M. Roumié, B. Nsouli, K. Zahraman, A. Reslan, Nucl. Instr. Meth. B 219-220 (2004) 389.

[9] B. Holländer, H. Heer, M. Wagener, H. Halling, S. Mantl, Nucl. Instrum. Meth. B 161-163 (2000) 227-230.

[10] J. L'Ecuyer, J.A. Davies, N. Matsunami, Nucl. Instr. Meth. 160 (1979) 337.

[11] H. Paul, J. Sacher, Fitted empirical reference cross sections for K-shell ionization by protons, At. Data Nucl. Data Tables 42 (1) (May 1989) 105-156.

[12] J.A. Maxwell, W.J. Teesdale, J.L. Campbell, Nucl. Instr. Meth. B 95 (1995) 407-421.

[13] N.P. Barradas, C. Jeynes, R.P. Webb, E. Wendler, Nucl. Instr. Meth B 194 (2002) 15.

[14] C. Jeynes, N.P. Barradas, E. SZilágyi, Anal. Chem. 84 (2012) 6061, http:// dx.doi.org/10.1021/ac300904c.

[15] U. Wätjen, H. Bax, P. Rietveld, Surf. Interface Anal. 19 (1992) 253.

[16] J.L. Campbell, T.L. Hopman, J.A. Maxwell, Z. Nejedly, Nucl. Instr. Meth. B 170 (2000) 193.

[17] J.L. Campbell, N.I. Boyd, N. Grassi, P. Bonnick, J.A. Maxwell, Nucl. Instr. Meth. B 268 (2010) 3356.

[18] P.C. Chaves, A. Taborda, N.P. Barradas, M.A. Reis, Nucl. Instr. Meth. B 268 (2010) 2010.

[19] M. Reis, IST/ITN, Universidade de Lisboa, private communication (2013). 